\title{
A Systematic Review on Unani Interventions in Type-2 Diabetes Mellitus
}

Itrat Malik' ${ }^{1 *}$, Sheikh Aziz Ahmed ${ }^{2}$, Tariq Nadeem Khan ${ }^{3}$, and Shariq shamsi ${ }^{4}$

${ }^{1}$ Department of Tahaffuzi wa Samaji Tib, National Institute of Unani Medicine, Bangalore, India

${ }^{2}$ Department of Pharmacology, National Institute of Unani Medicine, Bangalore, India

${ }^{3}$ Department of Kulliyat, National Institute of Unani Medicine, Bangalore, India

${ }^{4}$ Department of Unani Pharmacy, National Institute of Unani Medicine, Bangalore, India

*Corresponding author: Itrat Malik, Department of Tahaffuzi wa Samaji Tib, National Institute of Unani Medicine, Bangalore, India, Tel: +917411602926; E-mail: malik.itrat@gmail.com

Received September 25, 2018; Accepted October 05, 2018; Published October 10, 2018

\begin{abstract}
Background: The prevalence of diabetes is steadily increasing worldwide, and it is considered as one of the main threats to human health. In India, numerous reports describe the utilization of Unani medicine in the treatment of diabetes mellitus.

Objective: to systematically analyzed randomized clinical trials of Unani interventions in type-2 diabetes mellitus.

Materials and Methods: A comprehensive search of online databases, viz., Pub Med, Google Scholar etc. using keywords like 'Unani Medicine', 'Diabetes mellitus', 'Clinical trial', Glycemic control' or combination of these. In addition, journals and dissertations in the library of the National Institute of Unani Medicine, Bangalore, India was manually searched. Only four clinical trials met the criteria and were included in the review. The most common outcome measures encountered in these studies were fasting blood glucose level (FBS), postprandial blood glucose level (PPBS) and $\mathrm{HbA} 1_{\mathrm{C}}$. Trials on pre diabetes and those which primarily examined diabetic complications such as diabetic neuropathy or nephropathy were excluded.

Results: Significant reductions in FBS and PPBS were observed with the administration of combination of Gymnema Sylvester and Tinospora cardifolia. Highly significant effect on FBS $(p<0.003)$ and moderately significant on PPBS $(p<0.013)$ were observed with Spirulina. Significant effect on $\mathrm{HbA} 1 \mathrm{C}(\mathrm{p}<0.05)$ was observed with a polyherbal formulation containing Tinospora cardifolia, Bambusa bambos and Nelumbo nucifera. Similar effects were observed on FBS, PPBS and $\mathrm{HbA} 1$ with administration of a combination of Eugenia jambolana and Withania coagulans. These studies enrolled 190 participants and treatment duration ranged from 6 to 12 weeks.

Conclusion: Although the results showed statistically significant effects with Unani interventions; however, due to limited number of trials, any definite conclusions regarding their efficacy cannot be ascertained. Hence, further studies with more number of participants and of sufficient length needed to determine a relevant clinical effect to validate the use of these interventions in routine clinical practice.
\end{abstract}

Keywords: Diabetes Mellitus; Unani Medicine; Unani intervention

\section{Retraction Note:}

The article entitled "A Systematic Review on Unani Interventions in Type-2 Diabetes Mellitus" has been accepted for publication in the Journal of Alternative \& Integrative Medicine considering the statements provided in the article as personal opinion of the author which was found not having any conflict or biasness towards anything. As the article was a perspective one, information provided by the author was considered as an opinion to be expressed through publication.

Soon after the publication of the paper, we witnessed some serious concerns and many of them argued that the paper is a personal perspective and had not discussed any relevant ethical issue considered under the journal scope. Moreover, the paper is neither innovative nor thought provoking.

Publisher took decision to make the article online solely based on the reviewers suggestion which considered the article not but a personal opinion of the author. However, it is found that the article has some unavoidable mistakes and issues, therefore, being retracted from the journal. 